Instituto Internacional de Investigación y Desarrollo Tecnológico Educativo INDTEC, C.A.

DOI: https://doi.org/10.29394/Scientific.issn.2542-2987.2019.4.11.20.380-395

OAI-PMH: http://www.indteca.com/ojs/index.php/Revista Scientific/oai

Ensayo Original / Original Essay

\title{
La Gestión del Conocimiento en las Universidades experimentales de Venezuela
}

\author{
Autora: Zulay Del Carmen Herrera Abache \\ Universidad Latinoamericana y del Caribe, ULAC \\ herreraabache@gmail.com \\ Caracas, Venezuela \\ https://orcid.org/0000-0003-2685-3231
}

Resumen

El presente ensayo tiene como fin la reflexión sobre la forma de manejar el conocimiento en la educación universitaria considerando factores de la realidad como la dinámica del entorno interno de la universidad y externo propio del país, así como factores de orden técnico, científico y humanístico dentro del marco de una concepción holística. En adición a su valor práctico, se recopila información actualizada sobre la gestión de conocimiento (GC) a la par de que los analiza considerando las realidades de la educación en las universidades experimentales de Venezuela. Para tal fin se sustenta en el documento descrito por Morles, Medina y Álvarez (2003), sobre la educación superior en Venezuela informe 2002 a IESALC-UNESCO, en el cual radica propósito de la experimentalidad universitaria; de igual forma la innovación en la gestión del conocimiento en la Universidad Simón Rodríguez, a pesar de ella, tema abordado por Ponce (1998), donde se argumenta la innovación y actualización de la GC, y el análisis realizado por Briceño (2003), ¿Por qué fracasan los proyectos tecnológicos educativos en las universidades venezolanas?. Análisis de caso de la Universidad Simón Rodríguez, donde se fundamenta la relevancia del tema abordado.

Palabras clave: conocimiento; gestión educacional; universidad. 


\title{
The Management of Knowledge in the experimental Universities of Venezuela
}

\begin{abstract}
The purpose of this essay is to reflect on the way knowledge is handled in university education considering factors of reality such as the dynamics of the internal environment of the university and the country's own external environment, as well as technical, scientific and humanistic factors within of the framework of a holistic conception. In addition to its practical value, up-to-date information on knowledge management (KM) is compiled and analyzed by considering the realities of education in the experimental universities of Venezuela. For this purpose, it is based on the document described by Morles, Medina and Álvarez (2003), on higher education in Venezuela, report 2002 to IESALC-UNESCO, which is the purpose of university experimentalism; Likewise, innovation in knowledge management at Simón Rodríguez University, in spite of it, is an issue addressed by Ponce (1998), where the innovation and updating of CG is argued, and the analysis carried out by Briceño (2003), Why do educational technology projects fail in Venezuelan universities?. Analysis of a case: Universidad Simón Rodríguez, where the relevance of the topic addressed is based.
\end{abstract}

Keywords: knowledge; educational management; university. 


\section{Introducción}

Históricamente, la concepción de un modelo de universidad diferente al de las universidades tradicionales venezolanas, elitistas y predominantes hasta finales de la mitad del Siglo XX, teniendo sus orígenes hacia las década de los años cincuenta (50), por ello se establecieron los parámetros en la Ley de Universidades (1967a), en la cual se expresa que: "la Universidad es fundamentalmente una comunidad de intereses espirituales que reúne a profesores y estudiantes en la tarea de buscar la verdad y afianzar los valores trascendentales del hombre" (art. 1).

Esta Ley reformada introduce en su texto el concepto de universidad experimental, visto como una alternativa en relación a la tradicional universidad autónoma; el hecho de su creación aparece con la idea de presentar nuevas estrategias de enseñanza, considerándose como una necesidad histórica, ya que las tradicionales han sido resistentes a las transformaciones, aunado a la incrementación de y crecimiento matricular, la multiplicación de sus funciones y programas dictados y en consecuencia la facilitación de una gestión consciente, productiva y eficiencia, que garantizara una formación plena de los ciudadanos en consonancia con la contribución y correspondencia social de los problemas nacionales.

Aun cuando esta ley data de más de 40 años, la Constitución de la República Bolivariana de Venezuela (1999): señala que "se consagra la autonomía universitaria para planificar, organizar, elaborar y actualizar los programas de investigación, docencia y extensión" (art. 109); estableciendo a su vez que las Universidades Experimentales (UE) alcanzaran su autonomía de conformidad con la ley y hace énfasis en la función indeclinable del Estado en relación a la educación como instrumento del conocimiento científico, humanístico y tecnológico, por ende su relación estrecha con la gestión de conocimiento (GC).

Mucho se ha venido trabajando en la creación y actualización de los 
esquemas de gestión de conocimiento en la Universidades Experimentales, sin embargo, los proyectos se abandonan por una serie de aspectos relacionados con la forma de gestión el conocimiento. En este sentido, es importante mencionar el proyecto Ciencia-Tecnología-Sociedad (CTS), impulsado por el Vice Rectorado Académico de la Universidad Nacional Experimental "Simón Rodríguez" hacia finales de la década de 1980 y hasta principios de la década de 1990, según lo señalara Ponce (1998): "Culminó en el olvido y todo el esfuerzo creativo de un equipo interdisciplinario de docentes para las áreas de ciencia, educación ambiental y educación para la salud, se perdió por el abandono gradual de los objetivos de ese proyecto" (pág. 11).

De forma semejante, Briceño (2003a): destacó la inexistencia de un "modelo de gestión del conocimiento complementado con una cultura tecnológica, poco compromiso de la universidad para gestionar los cambios a consecuencia de la tecnología, barreras económicas o presupuestarias y el alejamiento de las universidades con las comunidades de aprendizaje" (pág. 48).

Uno de los aspectos que destaca Briceño (2003b): es la no consideración del factor entorno para el modelo de gestión del conocimiento, la resistencia al cambio y la falta de adopción, en el modelo, de la "concepción transdisciplinaria de la gerencia del saber" (pág. 48); gerencia ésta que, hasta la actualidad, ha estado signada por una tendencia mecanicista propia de los siglos XIX y mediados del XX, pero impropia para los retos que se plantean a partir del Siglo XXI en términos de una conciencia formada sobre el desarrollo sustentable y el reconocimiento de la otredad, en contra de las vetustas tendencias de imponer pensamientos únicos bajo el dogma de una supuesta felicidad suprema.

Como puede evidenciarse la gestión del conocimiento en las Universidades Experimentales Venezolanas, están alejadas en su totalidad de la disposición fundamental de la creación de estas, siendo que se plantea a la 
universidad como una comunidad de intereses comunes y en la gestión se excluye estos intereses.

En consecuencia, el aporte en este ensayo radicaría en dar a conocer los factores de la realidad como la dinámica del entorno interno de la universidad experimental venezolana y externo propio del país, así como factores de orden técnico, científico y humanístico dentro de la gestión del conocimiento.

\section{Desarrollo}

2.1. La Gestión de Conocimiento en las Universidades Experimentales Venezolana

Entre las innovaciones introducidas en el marco de la experimentalidad, para interpretar los planteamientos en el campo de las universidades experimentales venezolanas, con respecto a la gestión del conocimiento a partir de las políticas de la educación universitaria, la Universidad Nacional Experimental Simón Rodríguez (UNESR) incluyó las siguientes:

a. El estudiante no tenía restricciones en cuanto a tiempo y lugar de estudio y para ello se le ofrecían distintas modalidades de enseñanza aprendizaje, desde la acreditación por experiencia hasta los cursos en línea, sin excluir el sistema tradicional de clases presenciales.

b. El estudiante avanzaría, de acuerdo con sus capacidades y rendimiento académico demostrable.

c. El rendimiento sería verificado a través de un enfoque multidimensional de los procesos de evaluación y los aspectos que intervienen en la formación de los estudiantes.

Por medio de este programa se propuso permitir mayores libertades vivenciales que contrastaran, complementaran o superaran las disposiciones librescas a las que los estudiantes estaban acostumbrados. En este sentido, 
se observa que al estimular la capacidad crítica del estudiante, a través de la discusión en seminarios y reuniones de trabajo, éste aprehendería y estaría mejor preparado para enfrentarse a un mundo con cambios constantes que repercute en el avance del conocimiento de la ciencia y la tecnología.

Otro aspecto digno de destacar dentro de la visión experimental de la (UNESR), era que su modelo educativo se inspiraba en la capacidad de discernimiento, el análisis y la toma de decisiones que desarrolla una persona y que va en consonancia con el proceso de aprendizaje implantado; y a su vez, éste se fundamentaba en módulos instruccionales que orientaban al estudiante en la producción del conocimiento de forma sistemática y ordenada.

En este sentido se trata de un proyecto que de acuerdo con el fundamento teórico-filosófico de la UNESR (1976a): "tiene como propósito la transformación de la educación, basándose plenamente en la función social, desde lo económico, cultural, recreativo y axiológico" (pág. 48); es decir, que conllevan al logro de la autorrealización, la dignificación humana y el desarrollo de líneas de producción propia de ingresos que estarían destinados al autofinanciamiento.

En último lugar, tanto la (UNESR), como muchas otras universidades, entre ellas la universidad experimental Ezequiel Zamora, contribuyeron al establecimiento de las estaciones experimentales, teniendo como función la producción, como sustento propio para obtener y venderse excedentes 0 subproductos tales como café, ganado bovino, avicultura y productos derivados, legumbres y hortalizas, miel de abejas, entre otros; siendo esto parte de la gestión de conocimiento de las universidades experimentales en sus inicios.

\subsection{Características de las Universidades Experimentales en Venezuela}

Los criterios de las universidades experimentales venezolanas en los Modelos de Gestión del Conocimiento (MGC) presentan las siguientes 
características teóricas formuladas en los principios filosóficos de la UNESR (1976b):

a. Proponen un sistema flexible de estudios, con varias modalidades a escoger por el estudiante, tales como estudios supervisados, estudios presenciales, estudios en línea y acreditación por experiencia; b. La enseñanza y aprendizaje elementalmente es un principio andragógico; c. Se enlaza directamente con las comunidades al transferir conocimientos que permiten resolver problemas reales relacionados con la producción, la salud, la educación y otros problemas; d. Proponen carreras que, en principio, no estaban planificadas en las universidades tradicionales autónomas. Por ejemplo, Ingeniería de Alimentos en el Núcleo Canoabo de la (UNESR); e. Poseen un sistema de gestión de conocimiento que se nutre (en teoría) de la participación conjunta de aquellas comunidades que pertenecen al área de influencia de las Universidades Experimentales (UE), así como los docentes y estudiantes; quienes conforman de esta forma las comunidades de aprendizaje; f. El sistema de gestión de conocimiento, por ser experimental, es (también en teoría), flexible y abierto a la innovación; y g. El sistema de gestión de conocimiento está adecuado a las (TIC's) para reducir brechas tecnológicas definidas entre las universidades de las grandes ciudades y aquellas establecidas como núcleos satélites en la provincia (pág. 49).

No obstante, a pesar de que existen una serie de ventajas y argumentos descritos en los documentos de reglamentación y funcionamiento de las Universidades Experimentales; en la actualidad la misión de éstas se ha desviado sustancialmente.

Con relación a ello, Briceño (2003c), refiere que:

Uno de los aspectos que produce la desviación de la misión de las UE es la no consideración del factor entorno para el modelo de gestión de conocimiento, la resistencia al cambio y la falta de adopción en el modelo de la concepción transdisciplinaria de la gerencia del saber (pág. 48).

Con base en estos argumentos es importante destacar que la gerencia, 
ha estado signada a lo largo de los años por una tendencia mecanicista propia a los siglos XI y XX, pero impropia para los retos que se plantean a partir del Siglo XXI en términos de una conciencia formada sobre el desarrollo sustentable y el reconocimiento de la otredad, en contra de las vetustas tendencias de imponer pensamientos únicos basados en una gestión dogmática, que desvirtúa la verdadera gestión de conocimiento de las Universidades Experimentales en Venezuela y excluye a las comunidades de aprendizaje de cualquier método de participación abierta y protagónica.

\subsection{Noción Primigenia de la Experimentalidad Universitaria y la Idoneidad de los distintos Sistemas de Gestión de Conocimiento.}

De acuerdo con la Ley de Universidades (1967b):

El ejecutivo Nacional, podrá crear Universidades Nacionales Experimentales, con el fin de ensayar nuevas orientaciones y estructuras en Educación Superior. Estas universidades gozaran de autoridad dentro de las condiciones especiales requeridas por la experimentación educativa. Su organización y funcionamiento se establecerá por reglamento ejecutivo y serán objeto de evaluación periódica a los fines de aprovechar los resultados beneficiosos para la renovación del sistema y determinar la continuación, modificación o supresión de su estatus (art. 10).

En relación con ello, se establece que el término significó para ese entonces el perfil sustancial del subsistema universitario, aunado a la puesta en práctica del incremento de la población estudiantil.

Sin embargo, la noción primigenia de la experimentalidad en la realidad e idoneidad de los sistemas de gestión del conocimiento asociados a dicha noción colocan entredicho el modelo prevaleciente de gestión del conocimiento, por lo que brevemente se describen:

a. La experimentalidad fue mostrada en nuevos sistemas de gestión del aprendizaje y la enseñanza, así como en las áreas administrativas; la cual 
funcionó durante una etapa de consolidación de las universidades experimentales, mientras se redactaban los primeros reglamentos de funcionalidad de estas. A partir de éstos, que fueron tardíos a la concepción primigenia de la experimentalidad, la universidad experimental comenzó a perder su protagonismo, en su propósito con las comunidades y la gestión que garantizara una orientación del aprendizaje y evaluación de conocimientos distintos a la forma tradicionalmente establecida, que respondiera además a un fundamento cónsono, creándose estaciones experimentales, laboratorios y talleres que garantizaran las prácticas profesionales y que debían surgir de los productos de investigación, docencia y extensión.

b. En algunas universidades como es el caso demostrado de la (UNESR), se puede inferir que el modelo experimental se ha desarrollado bajo concepciones equivocadas, algunos sesgos, tergiversaciones y falsos conceptos de la experimentalidad. Por tanto, valiéndose de una búsqueda inexplicable e inexistente de la calidad académica se llevan a cabo proyectos personales, alejados del objeto institucional; es decir; se ha mal utilizado el carácter experimental, dejando de lado la verdadera función para lo cual fue creada, y se ha basado en la improvisación de modelos de gestión del conocimiento.

c. El ingreso y ascenso del personal docente no se cumple con relación a los criterios establecidos para tal fin, al igual que se obvia los criterios de formación docente, además ha sido objeto de modificación con base a criterios netamente políticos, aunque sin intención de cercenar la pluralidad de pensamientos; acción que se enmarca en parámetros de imposición.

En este orden de ideas se puede citar a Morles, Medina y Álvarez (2003), quienes describen que:

El sistema propuesto se fundamenta conceptualmente en tres elementos: la calidad universitaria; la evaluación como proceso 
de control y aseguramiento de la calidad universitaria y la evaluación como acreditación. Se destaca además en el diseño un mecanismo de evaluación que permite asegurar la calidad de las instituciones universitarias en tres fases: creación de instituciones y programas, seguimiento o supervisión y rendición de cuentas (pág. 24).

En efecto, se evidencia que el propósito por el cual fueron creadas las (UE), se aleja sustancialmente de la realidad existente, convirtiéndose la (GC) en objeto de imposiciones, apetencias personales y alejando toda posibilidad de gestionar el conocimiento, reconociendo a la comunidad de aprendizaje como parte del proceso, tal como lo expresa la Ley de Universidades.

\subsection{El Pacto Social y su Influencia en la Gestión de Conocimiento en las} U.E.

En la República Bolivariana de Venezuela, el pacto social está normado y descrito en la Constitución de la República Bolivariana de Venezuela (CRBV), las leyes, reglamentos y ordenanzas que no contradigan la carta magna. Se encuentra basado fundamentalmente en la paz, la administración de la justicia, los derechos humanos y la potestad inalienable de la libre elección de los gobernantes periódicamente por medio de la participación libre y protagónica a través de los mecanismos legalmente permitidos, entre ellos el ejercicio de la contraloría social.

Es decir; que la sociedad venezolana está cada vez más inmersa en la esfera del conocimiento y la globalización, por lo tanto; cobra valor y se capitaliza con la capacidad de ser, hacer, pensar, innovar por medio de la adquisición del conocimiento científico y tecnológico. Significa entonces que emerge una necesidad continua de educación y formación académica, donde interviene activamente el sistema de gestión de conocimiento en las universidades experimentales, a través de métodos innovadores para un aprendizaje real y significativo. 
En efecto, a través del pacto social, la necesidad de educación se satisface siguiendo un orden legal preestablecido. Es por lo que se deben cumplir varias etapas, desde la educación preescolar hasta la educación básica y diversificada, para ingresar a la universidad o cumplir con el requisito de licenciatura para optar a un post grado. De lo contrario se genera desorden, entropía y caos determinístico por errores en la fijación de las condiciones iniciales para tener acceso al sistema de gestión del conocimiento que opera en el nivel universitario.

Cabe señalar que, en ninguna de las fuentes consultadas sobre la gestión del conocimiento, sus autores han considerado el pacto social como factor influyente en la comunicación de orden para establecer el sistema de enseñanza y aprendizaje sistemático y cónsono con las necesidades de la sociedad. Por ello aquí es importante considerarlo por cuanto es un elemento regulador en los componentes del ambiente del sistema complejo de gestión de conocimiento-universidad experimental. Sería comparable con un modulador directo de la intensidad con la que se expresan las necesidades de preparación académica e indirecta de la dinámica en la gestión de conocimiento.

\subsection{Influencia del Entorno Ideológico actualmente sobre la Gestión de Conocimiento en la Universidad Experimental}

Es importante destacar que el estrato ideológico del entorno está estrechamente relacionado la noción, doctrina o ideología socioeconómica que intente permear hacia el interior del complejo sistémico o salir de él.

Para el momento existe una doctrina de Estado: el Socialismo del Siglo XXI que tiene como base ideológica la inclusión, la masificación, la educación liberadora, participativa y protagónica; por lo tanto, como respuesta y acompañamiento a las políticas educativas actuales, se crearon las Siguientes Universidades; Universidad Nacional Experimental de la Seguridad (UNES); 
Universidad Nacional Experimental Politécnica de la Fuerza Armada Bolivariana (UNEFA) y la Universidad Nacional Experimental Marítima del Caribe (UMC), entre otras; ya que algunas de las creadas anteriormente, por medio de su ideología y educación elitesca, se convirtieron en una barrera hacia la entrada de otras corrientes libres de pensamiento, menoscabando así lo que el Estado tiene establecido constitucionalmente.

Es evidente entonces, que en otras universidades fundadas como experimentales; tal es el caso de las Universidades Simón Bolívar y la Experimental del Táchira, por citar algunas, el estrato ideológico permea hacia el exterior la doctrina capitalista neoliberal, cuya política de ingreso sólo permite un grupo limitado de población estudiantil con unas características y cualidades pre establecida en el perfil de ingreso, con una posición ideológica no cónsona con las políticas del Estado Venezolano, centradas en la negatividad constante a los cambios ideológicos que potencialmente restringen la gestión de conocimiento, la creación de formas organizacionales que aprenden, además de las posibilidades de generar, integrar y conformar equipos con valores compartidos, con una concepción sistémica como elemento fundamental en la conformación de comunidades de aprendizajes que vayan más hacia un Modelo de Gestión de Conocimiento Holístico.

\section{Conclusiones}

En el contexto de las universidades experimentales venezolanas, la Gestión de Conocimiento aplica una respuesta positiva porque en cualquier institución universitaria su principal activo es el capital humano, que configura a su vez el capital intelectual. Por otra parte, es una organización con su estructura jerárquica, sus normas, su cultura, sus valores, su misión y visión; de igual modo es una empresa para producir tanto bienes materiales tangibles, traducidos en productos tecnológicos propios del proceso de investigación aplicada; como intangibles, en términos de conocimientos transferidos en un 
proceso de enseñanza-aprendizaje hacia individualidades (personas) que se licencian como profesionales de una rama específica del conocimiento.

El compartimiento Estado perteneciente al ambiente del complejo sistémico gestión del conocimiento-universidad experimental, es el financiamiento, es decir, el suministro de los recursos financieros necesarios para que todo el complejo funcione sin que existan mayores limitantes económicas. Se trata entonces de la transferencia programada de capitales hacia las universidades dentro de un cronograma específico, sin retardos, para que no sea necesario reprogramaciones o improvisaciones.

Cabe señalar que la gestión del conocimiento en las universidades experimentales tiene un costo en los rubros de gastos de personal (nómina), biblioteca, laboratorios, mantenimiento de las construcciones y bienes patrimoniales (campus), sistema de becas, viáticos y actualización del conocimiento para el capital humano que a su vez, es portador del capital intelectual.

Se dirá que esto hace referencia sobre la forma de gestionar el conocimiento en la educación universitaria, considerando factores de la realidad como la dinámica del entorno interno de la universidad experimental y externo propio del país, así como factores de orden técnico, científico y humanístico dentro del marco de una concepción holística, que permita implementar un modelo de gestión interactivo, donde la opinión de cada uno de sus miembros es tomado en consideración por la alta gerencia de la institución universitaria a la hora de implementar cambios que vayan a favor del desarrollo institucional.

No puede existir una sociedad del conocimiento en la que haya un divorcio entre la universidad, la sociedad, la empresa privada y el sector público. La labor de los gobiernos está en unir y reforzar los lazos entre estos cuatro compartimientos del sistema, en tanto que la misión de las universidades radica en crear y poner en práctica modelos de gestión de 
conocimiento en las que se enlace, por una parte, la formación del talento humano en función del desarrollo necesario para el país; y por otra, la comunidad como un todo, hacia la cual la universidad se debe y se compromete en coadyuvar a resolver sus problemas con base a un flujo de conocimientos en correspondencia biunívoca (doble flujo de entrada y salida).

Finamente de esta manera el liderazgo efectivo emerge como un modelo gerencial digno de ser puesto en práctica en el ámbito universitario venezolano, motivado a la necesidad de que este tipo de recinto mejore su funcionalidad administrativa y docente, con el firme propósito de ofrecerles a los actores que en ellas laboran, una excelente gestión de conocimiento.

\section{Referencias}

Briceño, M. (2003a,b,c). ¿Por qué fracasan los proyectos tecnológicos educativos en las universidades venezolanas? Análisis de un caso: Universidad Simón Rodríguez. Arbitraje, 1(2), 39-49, ISSN: 1690-3609. Recuperado de:

https://biblat.unam.mx/es/revista/arbitraje/articulo/por-que-fracasanlos-proyectos-tecnologicos-educativos-en-las-universidades-

venezolanas-analisis-de-un-caso-universidad-simon-rodriguez

Constitución de la República Bolivariana de Venezuela (1999). Gaceta Oficial de la República Bolivariana de Venezuela, $\mathbf{N}^{\circ} \mathbf{5 . 9 0 8 .}$ (Extraordinario), jueves 19 de febrero. Caracas, Venezuela: La Asamblea Nacional de la República Bolivariana de Venezuela. Recuperado de:

http://www.asambleanacional.gob.ve/documentos archivos/constitucio n-nacional-7.pdf

Ley de Universidades (1967a,b). Gaceta Oficial de la República de Venezuela Extraordinaria, $N^{\circ} 1.429$, de fecha 08 de septiembre de 1970. Ordinaria $\mathbf{N}^{\circ}$ 28.262, viernes 17 de febrero de 1967. Caracas, 
Venezuela: Consejo Nacional de Universidades.

Morles, V., Medina, E., \& Álvarez, N. (2003). La educación superior en

Venezuela informe 2002 a IESALC-UNESCO. Caracas, Venezuela: IESALC-UNESCO. Recuperado de:

http://unesdoc.unesco.org/images/0013/001315/131594s.pdf

Ponce, M. (1998). La innovación en la gestión del conocimiento en la Universidad Simón Rodríguez, a pesar de ella. Ponencia 3 del foro "Investigación, Extensión y Docencia en la Educación Superior". Caracas, Venezuela: Mimeo.

UNESR (1976a,b). Filosofía y estructura de la Universidad Simón Rodríguez. Informe presentado al Consejo Nacional de Universidades. Barinas, Venezuela: Universidad Nacional Experimental Simón Rodríguez. 


\section{Zulay Del Carmen Herrera Abache \\ e-mail: herreraabache@gmail.com}

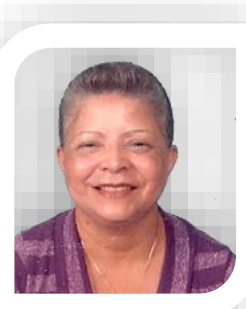

Nacida en Caracas, Distrito Capital, Venezuela.

Profesora de Ciencias Sociales; Especialidad Historia

y Geografía (Universidad Nacional Experimental

Libertador, UPEL - Instituto Pedagógico de Caracas);

Especialista de Educación Universitaria (Universidad

Santa María); Especialista en Gestión Pública

(Universidad Nacional Experimental Politécnica de la Fuerza Armada

Bolivariana, UNEFA); Magister Scientiarum en Educación Superior (Caribbean International University, CIU); Coordinadora Académica de los Programas Nacionales de Formación Avanzada Clínicos Hospitalarios, Universidad de las Ciencias de la Salud "Hugo Chávez Frías" año 2018.

El contenido de este manuscrito se difunde bajo una Licencia de Creative Commons ReconocimientoNoComercial-Compartirlgual 4.0 Internacional 